\title{
Design of an origami bendy straw for robotic multistable structures
}

\author{
Evandro Bernardes \\ Aix Marseille univ, CNRS, ISM, Marseille, France \\ Email: evandro.bernardes@univ-amu.fr \\ Stéphane Viollet* \\ Aix Marseille univ, CNRS, ISM, Marseille, France \\ Email: stephane.viollet@univ-amu.fr
}

\begin{abstract}
This paper presents a multistable soft cylindrical origami structure based on "bendy straws", consisting of multiple conical frusta. These frusta are connected in such a way that the whole structure is axially multistable, having a stable compressed state in which its smallest frustum is collapsed. The bendy straw structure can also be constructed in such a way that the smallest frustum can be only partially collapsed so as to keep the structure in the bent state. We also studied the geometry of a similar structure consisting of polygonal frusta instead of conical ones, and use this geometry to design a non-rigidly foldable origami pattern that folds into a similar origami bendy straw structure. Most of the origami structures presented so far are modeled from rigidly foldable origami patterns: origami structures that do not rely on deformation, but cannot use it to their advantage. Here we present a novel deformable (non-rigid) origami structure to which deformation/stress forces are applied to make it multistable. We established that this origami structure is not only axially multistable but that it can also be kept in a similar stable bent state by using Pop-Through Defects (PTDs). Multiple origami bendy straw cells are folded from $90 \mathrm{~g} / \mathrm{m}^{2}$ paper bi-laminated with a 42.5-micron thick plastic film. A digital dynamometer was used to study the forces required to compress and expand a single origami bendy straw cell, create and reverse a PTD, and bend an origami bendy straw using PTDs.
\end{abstract}

\section{Nomenclature \\ $R$ External radius of bendy straw structure. \\ $r$ Internal radius of bendy straw structure. \\ $\rho$ Ratio between external and internal radii. \\ $h_{1} \quad$ Height of bendy straw's top frustum. \\ $h_{2}$ Height of bendy straw's bottom frustum. \\ $\alpha_{1} \quad$ Angle of bendy straw's top frustum. \\ $\alpha_{2} \quad$ Angle of bendy straw's bottom frustum. \\ $n \quad$ Number of sides of polygon of origami bendy straw.}

*Address all correspondence to this author.
A Side length of external polygon of origami bendy straw. a Side length of external polygon of origami bendy straw.

$b_{1} \quad$ Height of flattened origami bendy straw pattern's top frustum.

$b_{2}$ Height of flattened origami bendy straw pattern's bottom frustum.

$\beta_{1} \quad$ Angle of flattened top frustum in the origami bendy straw origami pattern.

$\beta_{2} \quad$ Angle of flattened bottom frustum in the origami bendy straw origami pattern.

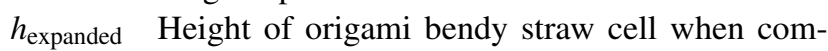
pletely expanded.

$h_{\text {middle }}$ Height of origami bendy straw cell when in the middle state.

$h_{\text {compressed }}$ Height of origami bendy straw cell when in the compressed state.

$\theta$ Bending angle.

$\theta_{\text {stable }}$ Bending angle in stable bent position.

$\theta_{\max }$ Max bending angle.

\section{Introduction}

In a recent Focus published in Science Robotics, Rus and Sung predict that origami robots will soon be developed for use as autonomous machines showing greater customizability and adaptability [1]. Origami-inspired structures can sometimes replace conventional mechanical assemblies. Origami structures can be quickly and easily prototyped with paper, and subsequently replacing paper with other materials gives these structures a durable life cycle, better shock absorption properties [2] and a low mass, while maintaining their flexibility without any need for conventional joints. Most of the origami patterns used so far have been rigidly foldable patterns/designs: they can be continuously folded even if all their facets are completely rigid. Rigidly foldable origamibased structures can be easily constructed with sheets of rigid material, and modeled, simulating the folds in the form of 
rotational hinges [3]. There also exist some non rigidly foldable origami patterns, but in order to make these models foldable, the material used has to be flexible and/or elastic enough for deformations to be possible during the folding process. Non rigidly foldable origami structures have some useful properties, and can adopt various mechanically stable states. Origami tubes are one particular class of 3D origami structures that hold great promise in fields such as medical robotics [4] and robotic manipulation as grippers [5]. Origami tubes have been thoroughly studied and modeled in recent studies $[6,7]$. Among these tubular structures, the Kresling tower is an origami-based structure of particular interest. This origami structure can change its height when a rotation is applied between its two extremities, converting a rotational motion into a translational one. Depending on the size of the folding angle, the Kresling structure can also be bistable, simultaneously showing both a tall standing state and a compressed low state [8]. Various assemblies of Kresling cells can also be multistable $[9,10]$ : the authors of several studies have described how to control the bistability of Kresling cells $[8,11]$.

It is worth noting that bistability and even multistability can also be observed in soft structures such as the common bendy straw, which can be either bistable or multistable as required [12]. This was the starting point of our study on the design and construction of a multistable origami tube. This bendy straw structure consisting of non-identical conical frusta can easily be made to become axially bistable, which means that it can adopt two different states: a tall stable state and a compressed state in which its smallest frustum is inverted. It is also possible to add another stable state: when the structure has some built-in stress, its frustum can become partially inverted, giving the structure a bent shape, the direction of which can be continuously varied while maintaining the bent state [12].

One of the advantages of origami structures over those based on bendy straws is that they can be constructed from folded sheets of thin material. Paper can be used, for example, for easy prototyping or artistic applications, or laminated paper to obtain greated rigidity while still being thin and flexible, as described in [13]. Origami structures can be easily produced in large numbers: laser-cutting is a much faster means of creating an origami pattern 3D printing or casting, as well as being more precise than 3D printing.

In this study, it was proposed to analyze the geometry of both the original bendy straw and a polygonal approximation of the bendy straw, and study for the first time an origami structure we have called the origami bendy straw. It is worth noting that this origami pattern is very similar to the previously presented REBO pattern [14], but we have described here how to introduce Pop-Through Defects (PTDs) in order to obtain the original bendy straw's bent position, which has never been applied to a REBO-like pattern so far. We have also established how to calculate the lengths and angles of each crease in the origami pattern in order to replicate a given bendy tower.

This paper focuses on the geometrical and design parameters required to construct an origami bendy straw structure

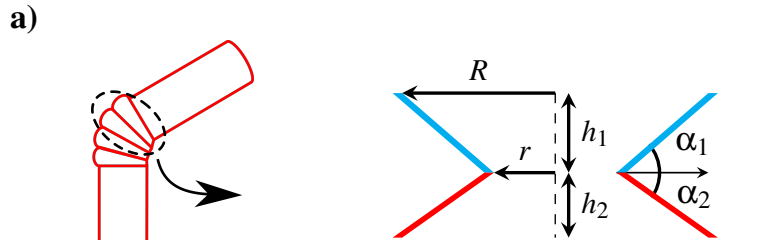

b)

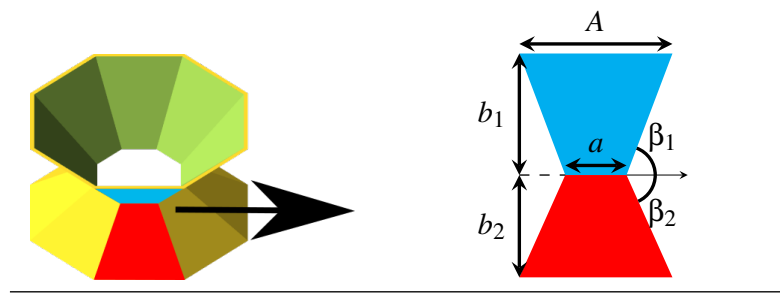

c)

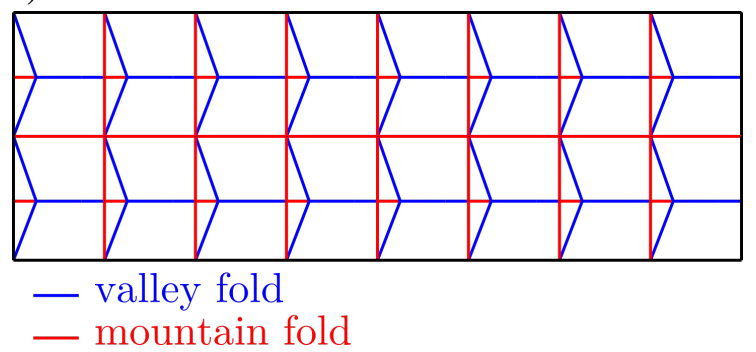

Fig. 1: a) Geometry of the original bendy straw structure. b) 3D model of an octogonal origami bendy straw and geometry of the top facets (in blue) and bottom facets (in red). c) Origami bendy straw pattern used to produce a similar structure/the same structure. Parameters: $\rho=0.5, n=5, \alpha_{1}=30$, $\alpha_{1}=20,2$ stages.

with any given height, radius and bending angle, while analyzing the conditions under which the structure is multistable. In the section 2, we describe the geometry of the bendy straw and introduce the origami bendy straw pattern. In the section 3 , the stress force required to compress and expand an origami bendy straw cell is analyzed. In the section 4, we show how to modify the creases of the origami in order to replicate the bent state of the bendy straw, while also analyzing the force required to bend it.

\section{Geometry and parametrization}

The original bendy straw cell has been described as a pair of conical frusta [12]. Bendy straws can be described in terms of the following parameters: $R$, the external radius of the cell, the ratio $\rho=\frac{r}{R}$ between the internal and external radii, and $\alpha_{1}$ and $\alpha_{2}$, the angles of the top and bottom conical frusta. As shown in Fig. 1a, $R, \rho, \alpha_{1}$ and $\alpha_{2}$ can be used to calculate $r$, the internal radius, $h_{1}$ and $h_{2}$, the heights of the 
a)

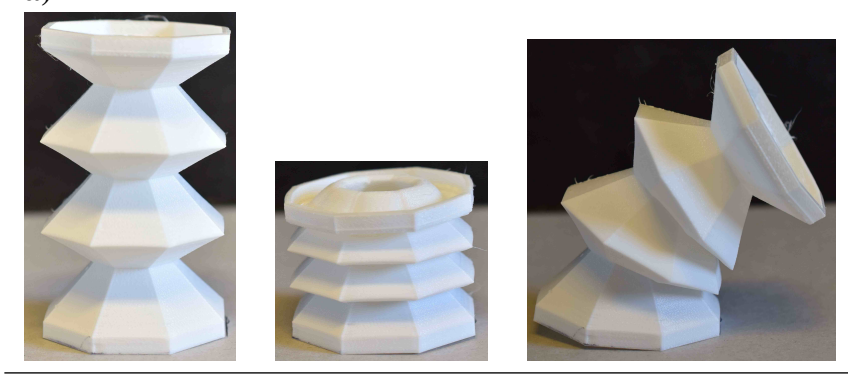

b)

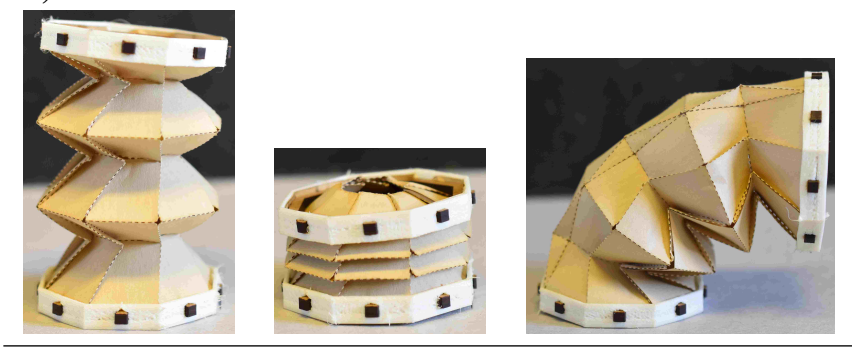

c)

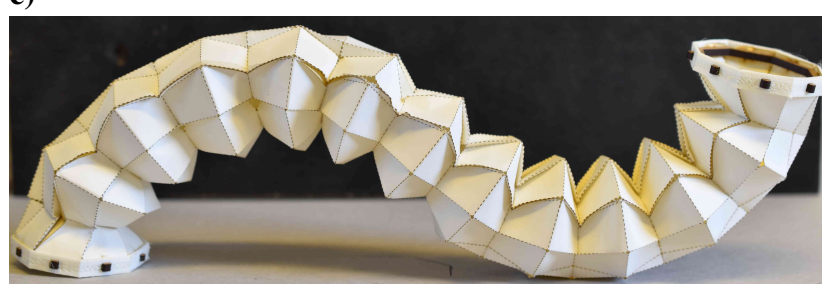

Fig. 2: a) Polygonal bendy straws 3D printed with Ultimaker White TPU. b) Origami bendy straw version of a similar structure. c) 12-stage origami bendy straw with an "S" shape. Half of the cells bend in one direction, and the other half in the opposite direction. Parameters: $R=25 \mathrm{~mm}, \rho=0.4$, $n=8, \alpha_{1}=45, \alpha_{1}=35$

two frusta as follows:

$$
\begin{cases}r & =\rho R \\ h_{1} & =(R-r) \tan \alpha_{1} \\ h_{2} & =(R-r) \tan \alpha_{2}\end{cases}
$$

A polygonal bendy straw structure can also be defined as a similar structure, the conical frusta of which are replaced by pyramidal frusta (truncated pyramids) having $n$ sides. To test the validity of this model, a 3D printer was used to obtain a 3 -stages polygonal bendy straw in Ultimaker White TPU (Fig. 2), with $R=25 \mathrm{~mm}, \rho=0.4, \alpha_{1}=45, \alpha_{2}=35$ (setting $\alpha_{1}-$ $\Delta \alpha=10), n=8$ and the thickness $t=2 \mathrm{~mm}$. The polygonal bendy straw structure was axially bistable right after being printed. After undergoing pressure in its compressed state for about 5 minutes (as explained in [12]), it continued to be stable in the bent position. As shown in the Fig. 1b, each frustum constituting the polygonal bendy straw structure is composed of multiple isosceles trapezoids. The angles and a)

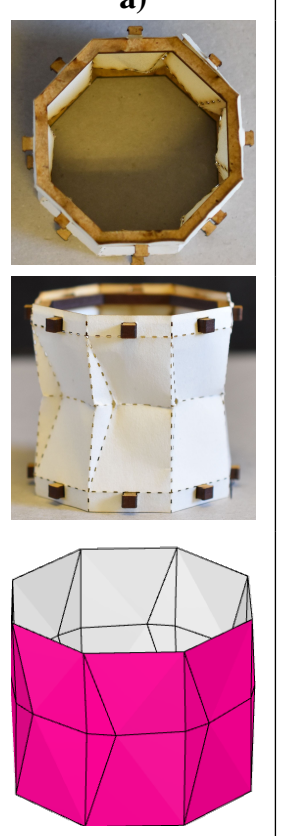

b)
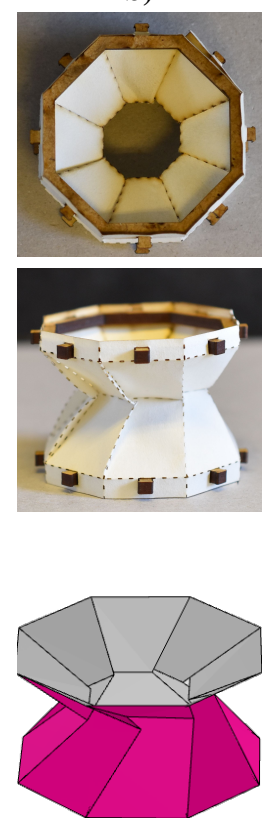

c)
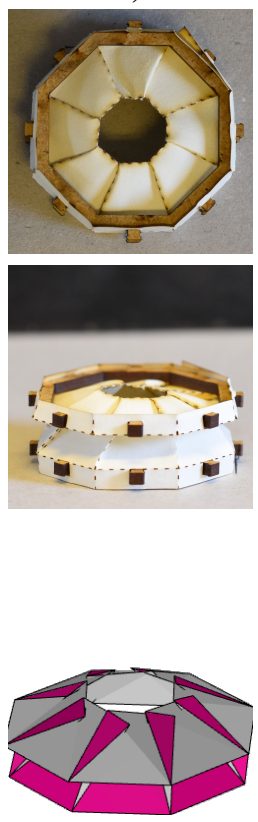

Fig. 3: Top view, side view and the simulation model for an origami bendy straw cell $(R=25 \mathrm{~mm}, \rho=0.4, n=8$, $\alpha_{1}=45, \alpha_{1}=35$ ). a) Completely deployed. b) Middle position. c) Completely compressed, with its top (smaller) frustum reversed.

dimensions of each trapezoid were calculated as follows:

$$
\left\{\begin{array}{l}
A=2 R \sin \frac{\pi}{n} \\
a=\rho A \\
\beta_{1}=\cos ^{-1}\left(\cos \alpha_{1} \sin \frac{\pi}{n}\right) \\
\beta_{2}=\cos ^{-1}\left(\cos \alpha_{2} \sin \frac{\pi}{n}\right) \\
b_{1}=(R-r) \frac{\sin \beta_{1}}{\cos \alpha_{1}} \\
b_{2}=(R-r) \frac{\sin \beta_{2}}{\cos \alpha_{2}}
\end{array}\right.
$$

Using the parameters defined in the equation 2 , the origami pattern shown in Fig. 1c was constructed. The vertices of the origami bendy straw pattern meet the conditions for flat foldability only when $\beta_{1}=\beta_{2}$, which is equivalent to $\alpha_{1}=\alpha_{2}$. For the fully compressed and collapsed state to exist, $\alpha_{1} \neq \alpha_{2}$ is therefore a necessary condition. Otherwise, compressing the tower will flatten instead of collapsing one of the frusta.

We observed that the behaviour of a single origami bendy straw cell with the parameters $(25 \mathrm{~mm}, 0.4,8,45,35)$ was similar to that of the original bendy straw. This was tested both by using an Origami Simulator ( $[15],[16])$ and by handfolding an origami bendy straw using regular/normal $90 \mathrm{~g} / \mathrm{m}^{2}$ paper bi-laminated with a 42.5-micron plastic film, on which the pattern was engraved by means of a Trotec Speedy 100 laser-cut machine. Examples of both procedures can be seen in Fig. 3. The extremities of the cell were fixed to a rigid polygonal support made of wood. These boundaries have to 
be constrained in order to ensure multistability. This structure was also found to be axially bistable, which indicates that bistability does not necessarily depend on the elasticity of the material. The height in each state can easily calculated. In the middle position, the height $h_{\text {middle }}$ was defined as follows:

$$
h_{\text {middle }}=h_{1}+h_{2}
$$

In the case of axially bistable origami bendy straws, it was observed that the smallest frustum was fully reversed in the compressed position, as expected to occur in the case of the original bendy straw. This assumption does not always hold true as can seen from the insert in Fig. 4c, where only part of the frustum is turned inside out, but when it does hold, the compressed state $h_{\text {compressed }}$ (where $h_{2}<h_{1}$ ) can be calculated as follows:

$$
h_{\text {compressed }}=h_{1}-h_{2}
$$

Contrary to what occurs with the original bendy straw, there is also a third fully unfolded state, the height of which was denoted $h_{\text {expanded }}$ which can be defined as follows:

$$
h_{\text {expanded }}=b_{1}+b_{2}
$$

The measurements of the heights of several towers were compared with the expected heights given by the equations (3) to (5), as shown in the Fig. 4. Only small errors were observed between the predicted and measured heights is small of the towers in the unfolded state (mean error $\mu=-0.61 \mathrm{~mm}$, standard deviation $\sigma=0.17 \mathrm{~mm}$ ) and the middle state $(\mu=2.15 \mathrm{~mm}, \sigma=2.29 \mathrm{~m})$. The largest error was obtained on the compressed state $(\mu=6.71 \mathrm{~mm}, \sigma=7.23 \mathrm{~m})$, especially with the tallest tower (see Fig. 4c). This error was due to the partial reversal of the state of the bottom frusta. This was observed with the largest values of $\alpha_{1}$ and $\alpha_{2}$ (which are both higher than 50), which resulted in higher measurement errors in the height of the structure in the compressed state.

\section{Force study}

A test bench was set up in order to analyze the forces exerted on the origami structure during its compression and expansion (Fig. 5). All the origami structures tested on this force bench were made of regular $90 \mathrm{~g} / \mathrm{m}^{2}$ paper bi-laminated with a 42.5-micron plastic film. The bench consisted of a motorized translation stage (Igus DLE-SA-0001 driven by a Gecko micro-step driver) and a digital dynamometer measuring the force (Sauter FH-20 digital). The origami structure was expanded and compressed in of $0.027 \mathrm{~mm}$ steps, and the forces were measured with a resolution of $0.01 \mathrm{~N}$.

Figure 6 shows the expansion/compression force curves recorded with several origami bendy straws and the heights predicted based on the equations (5), (3) and (4). Both curves had very similar shapes featuring a hysteresis, but upon analyzing the absolute values of the forces, the expansion and compression behaviour of the towers was found to differ considerably.

Figure 4 and Fig. 6 were compared in order to determine why the towers did not all adopt a stable compressed state. The origami bendy straw cell with the parameters ( $\left.\rho=0.7, n=8, \alpha_{1}=60, \alpha_{2}=50\right)$ shown in Fig. 4 did not reach a stable compressed state, and it can be seen from Fig. $6 \mathrm{~d}$ that the first force peak recorded with any similar tower was always negative. The origami bendy straw with the parameters $(25 \mathrm{~mm}, 0.5,8,30,20)$ shown Fig. 4 did not remain stable either in the compressed state. The largest version of the tower, having the parameters $(40 \mathrm{~mm}, 0.5,8,30,20)$, shown in Fig. $6 \mathrm{~b}$ did reach this state, but it was not very stable: simply touching the structure could make it "pop" into another more stable state. The Fig. $6 \mathrm{~b}$ clearly shows that the force corresponding to the first peak remained positive only very briefly.

When a fully compressed tower was expanded by hand beyond its first force peak, (between the first two red dots on Fig. 6), the tower quickly "snapped" into the next stable position, releasing the energy stored in the inverted frustum. We then kept on expanding the tower further but it did not show the same sudden response, which means that the tower must be/have been actively expanded until it was fully deployed. Trying to expand the tower even further would only have damaged the origami structure.

This behavior was not observed when compressing a fully expanded origami bendy straw structure: the fact that the force never changed its direction indicates that a constant compression force was required in order to compress the structure (the "snapping" movement that jumped it into the next state was weaker in this direction).

\section{Multistability with pop-through defects}

The origami bendy straw cell is axially stable, but does not naturally stay in a bent position. The original bendy straw can become multistable when built-in stress is introduced into the structure, as described by [12]. We could not use any of these methods to introduce multistability into an origami bendy straw because it requires the use of elastic material. One way of putting the origami bendy straw into a new stable position was to change the folding/folding pattern of some of the creases.

We used a mechanism named pop-through defect (PTD) [17]/A pop-through defect (PTD) procedure [17] was used here: by applying pressure to some of the vertices of the structure until it inverted its folds, popping into a different mechanically stable state. By using a PDT to "program" the state of the material, some parts of the structure we can made rigid as required. Adding a PDT to one side of an origami bendy straw (as in the Fig. 7) makes it stiffer and keeps it in an unfolded position. Instead of being compressed, the origami bendy straw bends to the opposite side. By choosing where to put the PDTs, we can create more complex structures (see 
a)

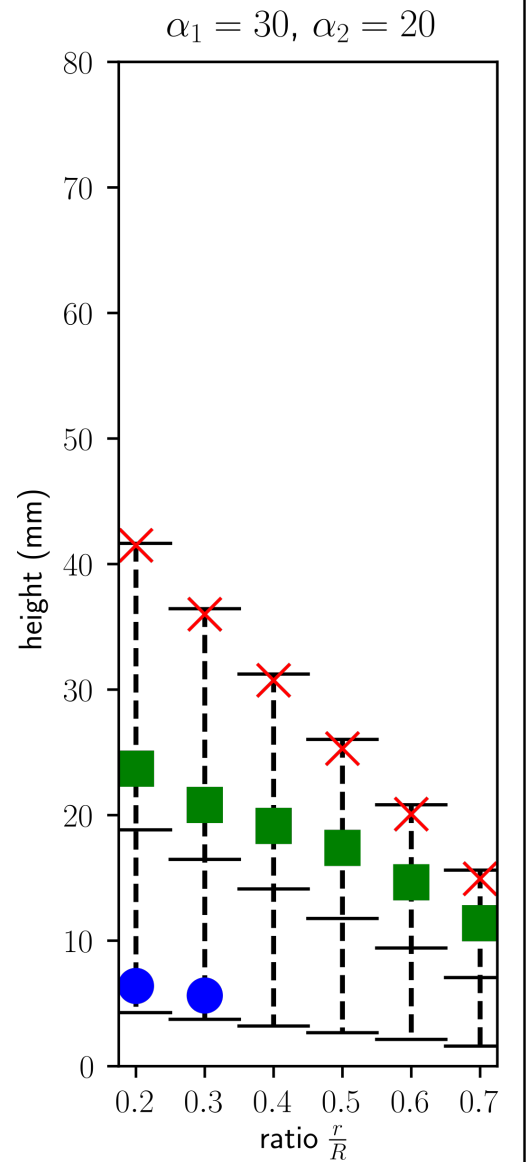

b)

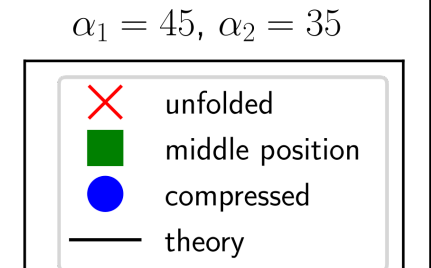

C)

$\alpha_{1}=60, \alpha_{2}=50$

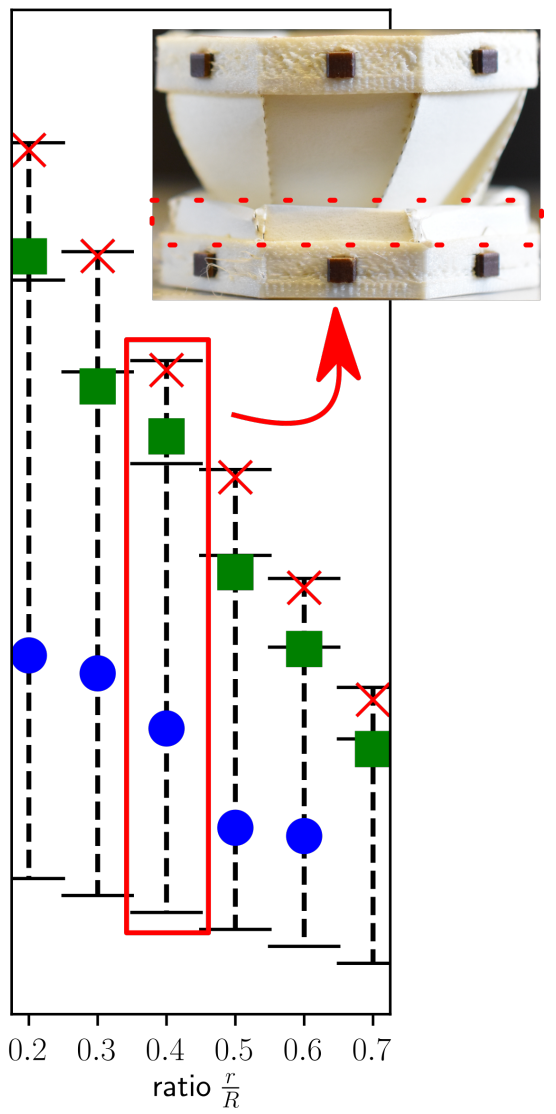

Fig. 4: Comparison between predicted and measured heights of various sets of towers with $n=8, R=25 \mathrm{~mm}, \Delta \alpha=10$ and $\rho$ ranging from 0.2 to 0.7 , with the following parameters: a) $\alpha_{1}=30$, b) $\alpha_{1}=45$ and c) $\alpha_{1}=60$. It is worh noting that how small values of heights make it impossible for the compressed stable state to exist. We can also note the large difference between height measurements of the towers in the compressed state with $\alpha_{1}=60$. This occurs because the smallest frustum is not completely inverted, as can be seen in the insert.

the Fig. 2c).

Our radially oriented paper origami bendy straw structure was then installed on the force bench, and a force of about $1.5 \mathrm{~N}$ was found to be required to either either make the vertex pop into a PTD or reverse it back into its original position. In the Figures $7 \mathbf{a}$ and $\mathbf{b}$, comparison are made between the crease assignments observed before and after creating a PTD: the Fig. $7 \mathrm{c}$ shows the force produced during this process.

\subsection{Bending angle}

It was tricky to find an analytical expression for $\theta_{\text {stable, }}$, the angle of the stable bent position, but the maximum bending angle $\theta_{\max }$ could be calculated (as in the Fig. 8):

$$
\cos \left(\theta_{\max }\right)=1-\frac{\left(b_{1}+b_{2}\right)^{2}-\Delta l^{2}}{2 L(L+\Delta l)}
$$

With even-numbered $n$ :

$$
\begin{cases}\Delta l & =\left|b_{1}-b_{2}\right| \\ L & =2 R \cos (\pi / n)\end{cases}
$$

And with odd-numbered $n$ :

$$
\begin{cases}\Delta l & =(R-r)\left|\frac{1}{\cos \left(\alpha_{1}\right)}-\frac{1}{\cos \left(\alpha_{2}\right)}\right| \\ L & =R(1+\cos (\pi / n))\end{cases}
$$

It is worth noting that the equation 6 is always independent of $R$ (both the nominator and the denominator are proportional to $R^{2}$ ). In addition, $\Delta l$ depends on $\alpha_{1}-\alpha_{2}$. As this difference becomes smaller, $\Delta l$ becomes negligible.

The Fig. 9 shows the measured stable and maximum $\theta$ recorded in the case of several different origami bendy straw cells. The measurements of the $\theta_{\max }$ matched the predictions quite closely. In addition, we noticed that the relationship 


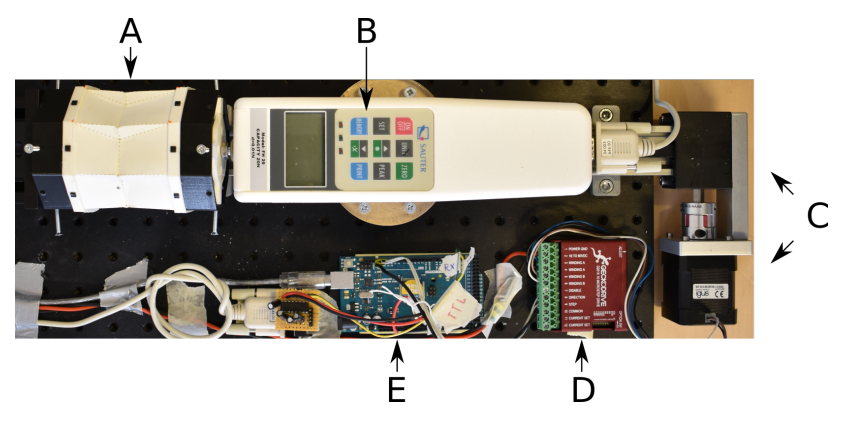

Fig. 5: The low-cost custom-made test bench used to measure the forces applied to the origami structure in (A). The force bench consisted of: a Sauter FH-20 digital force gauge (B), an Igus DLE-SA-0001 linear actuator $(\mathbf{C})$ driven by a Gecko micro-step driver (D), and an Arduino Mega (E) used to control the motor driver and deliver the measurements from the force gauge to a computer. With this force bench, we expanded and compressed the origami structure in $0.027 \mathrm{~mm}$ steps, while measuring the forces with a $0.01 \mathrm{~N}$ resolution.

between $\theta_{\text {stable }}$ and $\theta_{\max }$ depended on $\alpha_{1}$ and $\alpha_{2}$. For example, it was deduced from our curves that $\alpha_{1}=45$ and $\alpha_{2}=35$, from which it was concluded that that $\theta_{\max } \approx 2 \theta_{\text {stable }}$.

\subsubsection{Using two equal frusta}

In the case of applications that do not require the compressed state, we can set $\alpha=\alpha_{1}=\alpha_{2}$. The tower will not have a stable compressed state in this case, since instead of having an inverted frustum, the origami bendy straw cell will become flat-foldable. In this case, $\Delta l=0$ in both the even and odd cases, and we can show that:

$$
\sin \left(\theta_{\max } / 2\right)=\frac{1-\rho}{2 \cos (\pi / n)} \sqrt{\tan ^{2}(\alpha)+\cos ^{2}(\pi / n)}, \text { for even } n
$$

$\sin \left(\theta_{\max } / 2\right)=\frac{1-\rho}{1+\cos (\pi / n)} \sqrt{\tan ^{2}(\alpha)+\cos ^{2}(\pi / n)}$, for odd $n$

This equation was used to find the $\rho$ required to obtain a given set of $\alpha, \theta$ and $n$.

To study the force required to bend an origami bendy straw cell, we designed and produced a mechanical support consisting of a sliding joint and a ball joint. This support was connected to the tip of the force gauge and to one of the ends of an origami bendy straw, and the latter was then installed $2.5 \mathrm{~cm}$ to the right so that the force was applied offcenter. While measuring the force, a camera mounted on top of the test bench recorded the whole test. This video was then analyzed with Physlets Tracker [18] in order to relate the bending angle of the tower (Fig. 10). The stable position was found to match one of the zeroes of the expansion curve, and the maximum bending angle was found to be 35 , as predicted by the equation 6 .

\section{Summary}

Non rigidly foldable origami patterns are rather complicated to analyze, but they also promise to have some useful properties that are worth exploring. The origami pattern used here in a bendy straw structure/We introduced in this paper an origami pattern that replicates the bendy straw structure and could be used for many robotic applications. The possibility of constructing several bendy straws structures is liable to be necessary for prototyping future applications, since the large number of parameters involved makes it difficult to predict all the properties of these structures without testing them. The present origami version of the bendy straw takes much less time to produce than large number of 3D printed bendy straws or a single large bendy straw. In addition, PTDs provide a useful means of putting an origami bendy straw structure in a new bent state that would otherwise not be stable. Thanks to the use of PTDs, multistability and adjustable stiffness can be obtained much more easily than the built-in stress method based on the use of TPU material to make soft bendy straws. In future studies, it is planned to investigate how PTDs may serve to develop actuators such as shape-memory alloy actuators embedded in a structure making it possible to change its state dynamically as required.

\section{Acknowledgments}

We would like to thank J. Diperi and J-M Ingargiola for their technical assistance. This research was supported by CNRS, Aix-Marseille University and the French National Research Agency (ANR) via the Origabot project (ANR-18CE33-0008-01).

\section{References}

[1] Rus, D., and Sung, C., 2018. "Spotlight on origami robots". Science Robotics, 3(15), pp. 2-4.

[2] Sareh, P., Chermprayong, P., Emmanuelli, M., Nadeem, H., and Kovac, M., 2018. "Rotorigami: A rotary origami protective system for robotic rotorcraft". Science Robotics, 3(22), p. eaah5228.

[3] Demaine, E. D., and O'Rourke, J., 2007. Geometric folding algorithms: Linkages, origami, polyhedra, Vol. 9780521857.

[4] Cianchetti, M., Ranzani, T., Gerboni, G., Falco, I. D., Laschi, C., Member, S., and Menciassi, A., 2013. "STIFF-FLOP Surgical Manipulator mechanical design and". 2013 IEEE/RSJ International Conference on Intelligent Robots and Systems (IROS), pp. 3576-3581.

[5] Li, S., Stampfli, J. J., Xu, H. J., Malkin, E., Diaz, E. V., Rus, D., and Wood, R. J., 2019. "A Vacuum-driven Origami “ Magic-ball " Soft Gripper”.

[6] Filipov, E. T., Tachi, T., Paulino, G. H., and Weitz, D. A., 2015. "Origami tubes assembled into stiff, yet reconfigurable structures and metamaterials". Proceedings of the National Academy of Sciences of the United States of America, 112(40), pp. 12321-12326.

[7] Filipov, E. T., Paulino, G. H., and Tachi, T., 2016. "Origami tubes with reconfigurable polygonal cross- 
a)

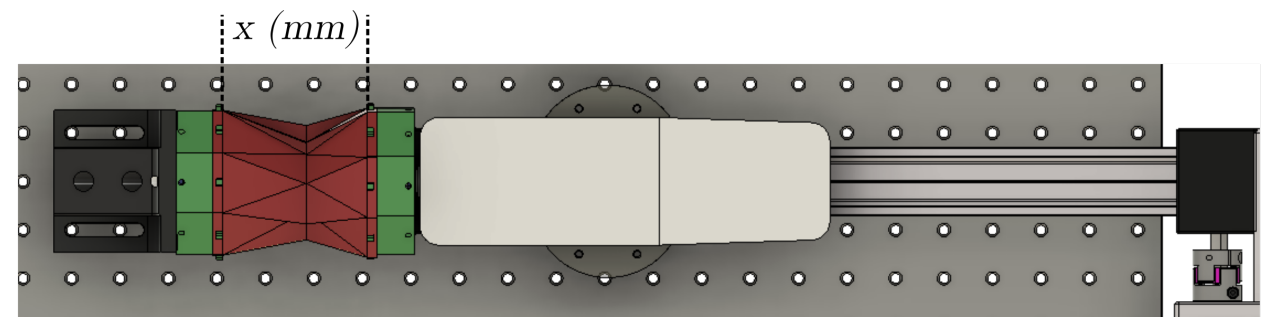

b)

$R=40 m m, \rho=0.5, \alpha_{1}=30, \alpha_{2}=20$
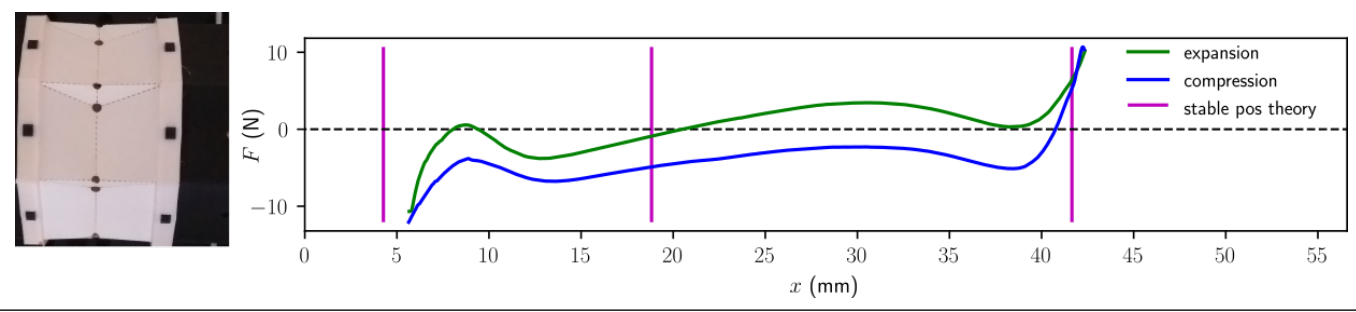

c)

$R=40 m m, \rho=0.5, \alpha_{1}=45, \alpha_{2}=30$

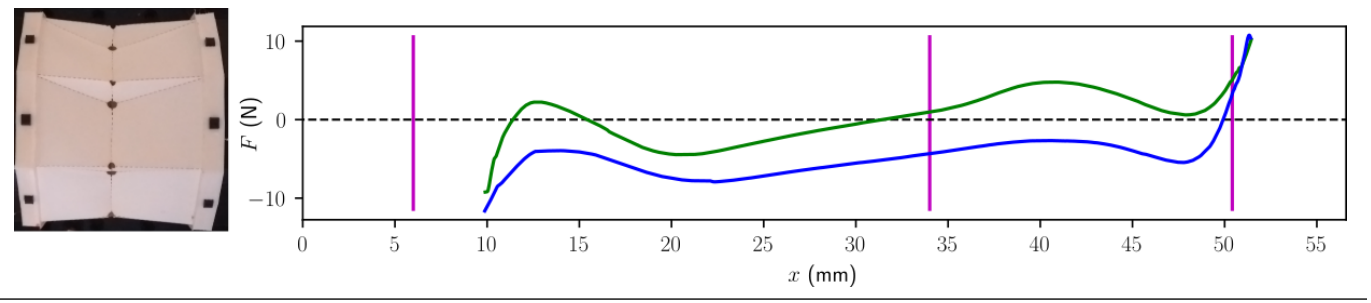

d)

$R=40 \mathrm{~mm}, \rho=0.7, \alpha_{1}=60, \alpha_{2}=50$
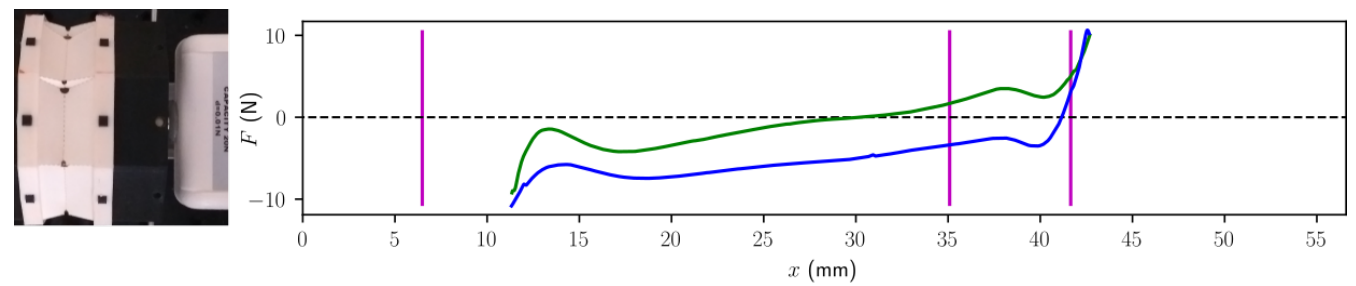

Fig. 6: a) CAD of test bench and an origami bendy straw structure $\left(R=40 \mathrm{~mm}, \rho=0.4, n=8, \alpha_{1}=45, \alpha_{1}=35\right)$. b-d) Force curves corresponding to the expansion (green) and compression (blue) of various origami bendy straws. Purple lines give the heights of the structure in the stable position predicted by equations 4,3 and 5 .

sections". Proceedings of the Royal Society A: Mathematical, Physical and Engineering Sciences, 472(2185).

[8] Zhang, Q., 2019. "Bistable behaviour of a deployable cylinder with Kresling pattern Bistable behaviour of a deployable cylinder with Kresling pattern".

[9] Pagano, A., Leung, B., Chien, B., Yan, T., Wissa, A., and Tawfick, S., 2016. "Multi-Stable Origami Structure for Crawling Locomotion". p. V002T06A005.

[10] Pagano, A., Yan, T., Chien, B., Wissa, A., and Tawfick, S., 2017. "A crawling robot driven by multi-stable origami”. Smart Materials and Structures, 26(9).
[11] Kidambi, N., and Wang, K. W., 2020. "Dynamics of Kresling origami deployment". Physical Review E, 101(6), p. 63003.

[12] Bende, N. P., Yu, T., Corbin, N. A., Dias, M. A., Santangelo, C. D., Hanna, J. A., and Hayward, R. C., 2018. "Overcurvature induced multistability of linked conical frusta: How a 'bendy straw' holds its shape". Soft Matter, 14(42), pp. 8636-8642.

[13] Chillara, V. S. C., Headings, L. M., and Dapino, M. J., 2015. "Self-folding laminated composites for smart origami structures". ASME 2015 Conference on Smart 
a)
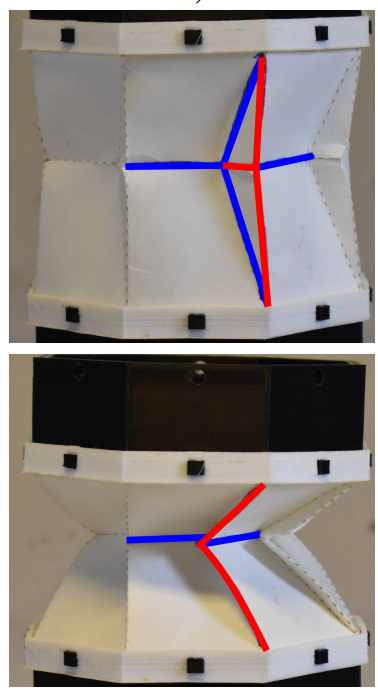

c)
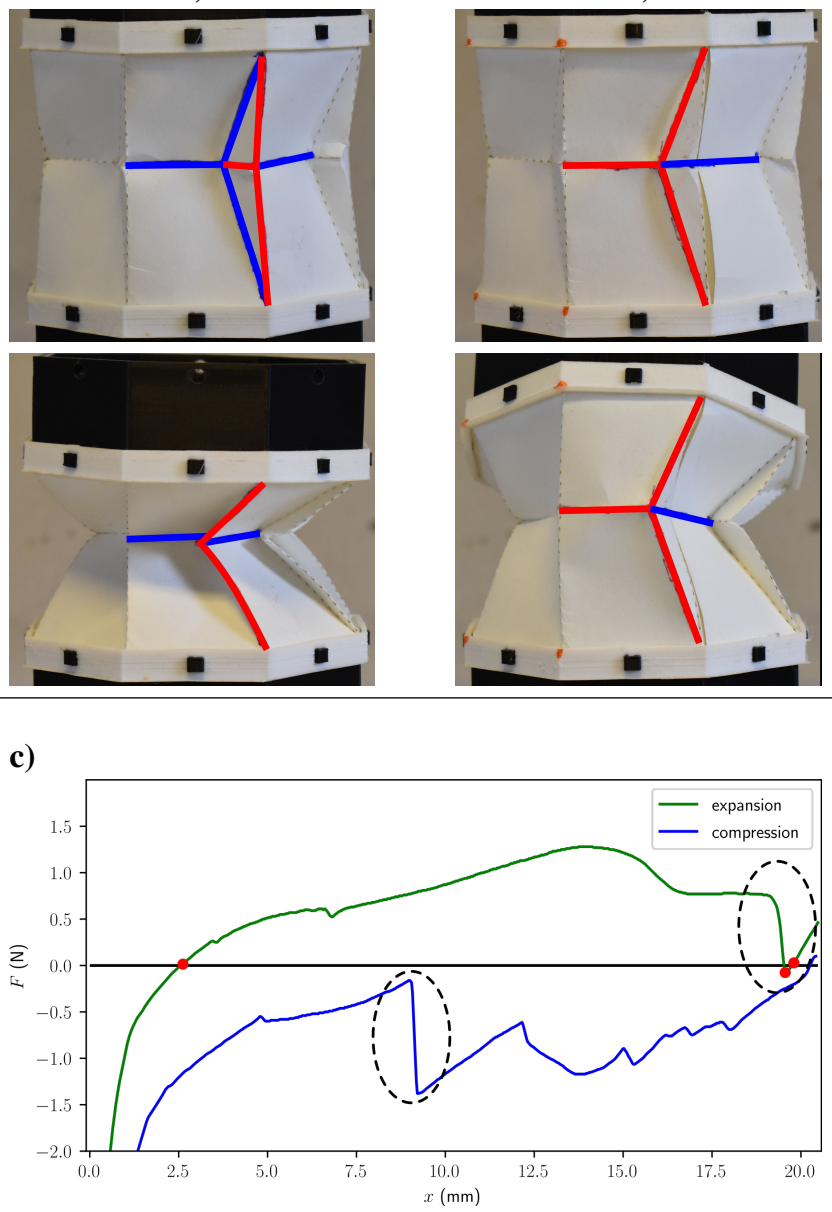

( a) $40 \mathrm{~mm}, \rho=0.4, \alpha_{1}=50$ and $\alpha_{2}=35$ forced to reach the maximum bending angle. b) Simplified geometry of the maximum bending angle. c-e) Various origami bendy straws produced with $R=25 \mathrm{~mm}$, showing how many cells are required to obtain a 180 bent tower. c) $n=6, \rho=0.4558$, $\alpha_{1}=\alpha_{2}=25$, producing cells with $\theta=36$. d) $n=7$, $\rho=0.3624, \alpha_{1}=\alpha_{2}=35$, producing cells with $\theta_{\max }=45$.

345(6197), pp. 647-650.

[18] Tracker, P. https://physlets.org/tracker/. accessed: 202102-08.
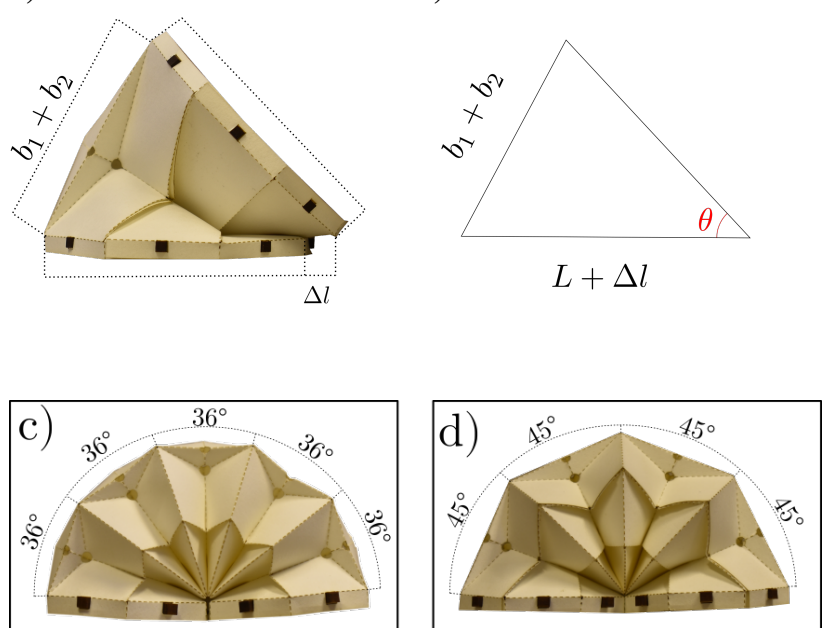

Fig. 7: Changes in the Mountain-Valley assignment: a) original origami bendy straw, and b) after creating a PTD. c) Force necessary to compress/expand the vertex of the laminated paper origami bendy straw and to pop it from one state to another. Parameters: $R=40 \mathrm{~mm}, \rho=0.4, n=8, \alpha_{1}=45$, and $\alpha_{1}=35$.

Materials, Adaptive Structures and Intelligent Systems, SMASIS 2015, 2(May 2018).

[14] Carlson, J., Friedman, J., Kim, C., and Sung, C., 2020. "REBOund: Untethered Origami Jumping Robot with Controllable Jump Height". Proceedings - IEEE International Conference on Robotics and Automation, pp. 10089-10095.

[15] Ghassaei, A., Demaine, E. D., and Gershenfeld, N., 2018. "Fast, Interactive Origami Simulation using GPU Computation". Origami ${ }^{7}$ : Proceedings of the 7th International Meeting on Origami in Science, Mathematics and Education (OSME 2018).

[16] Simulator, O. https://origamisimulator.org/. accessed: 2021-01-12.

[17] Silverberg, J. L., Evans, A. A., McLeod, L., Hayward, R. C., Hull, T., Santangelo, C. D., and Cohen, I., 2014. "Using origami design principles to fold reprogrammable mechanical metamaterials". Science, 


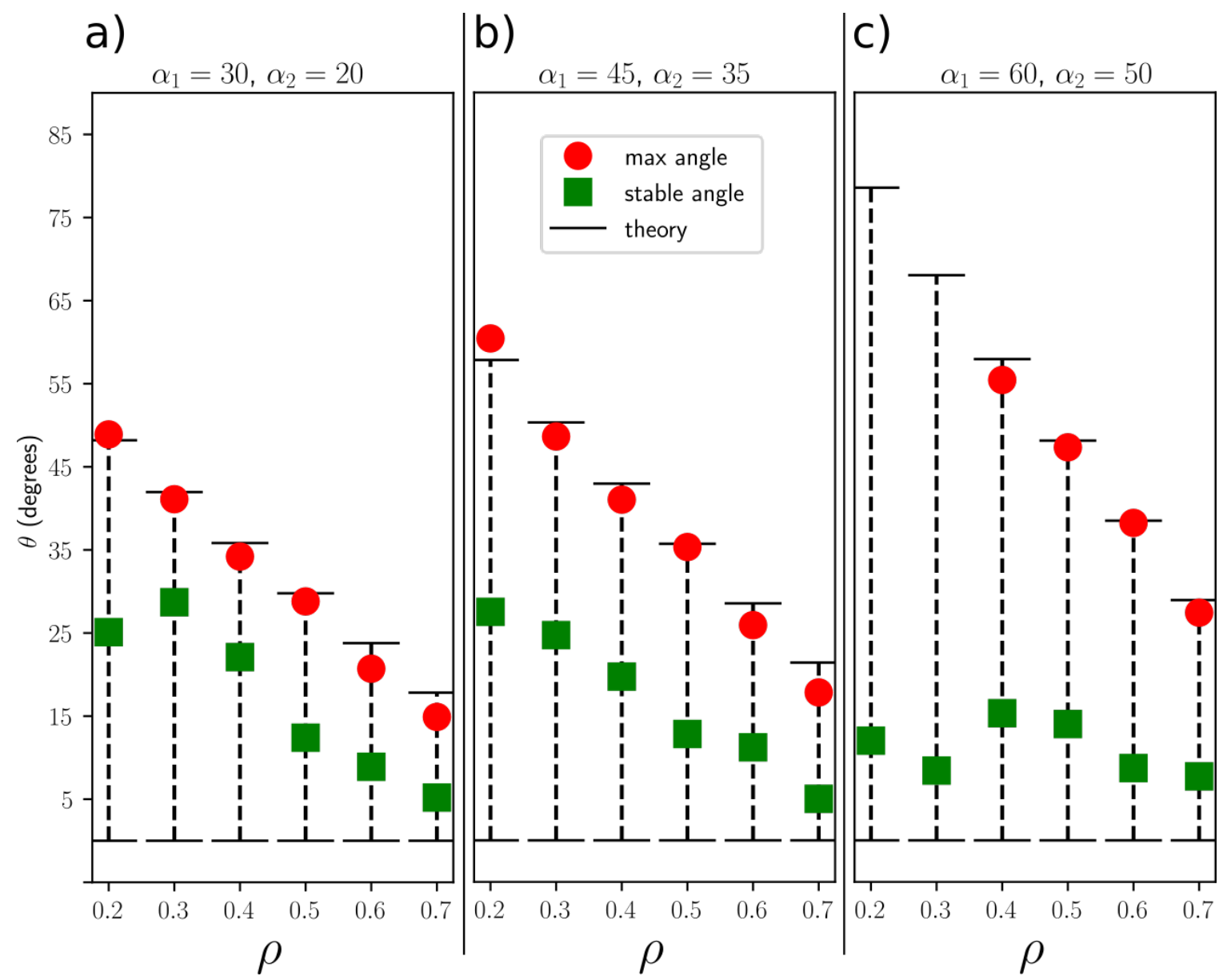

Fig. 9: Measured stable and maximum bending angles in various sets of towers with $n=8, R=25 m m, \Delta \alpha=10$ and $\rho$ ranging from 0.2 to 0.7 , with the following parameters: a) $\alpha_{1}=30$, b) $\alpha_{1}=45$ and c) $\alpha_{1}=60$. 
a)
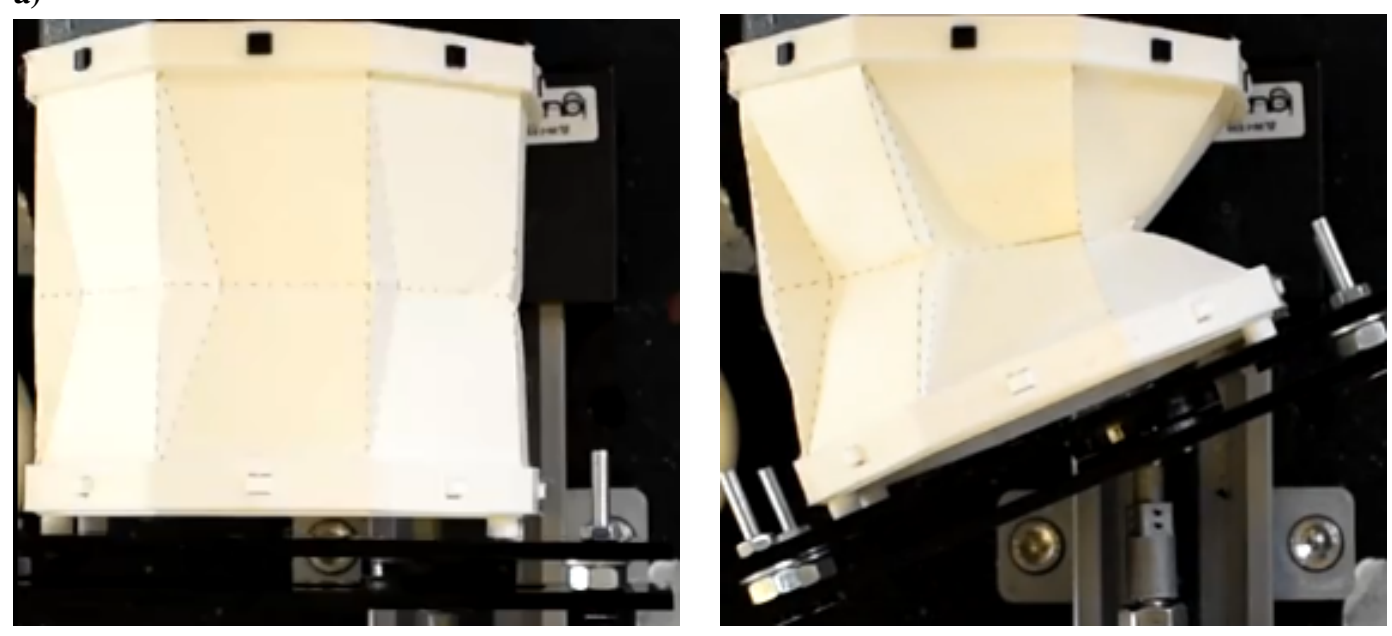

b)

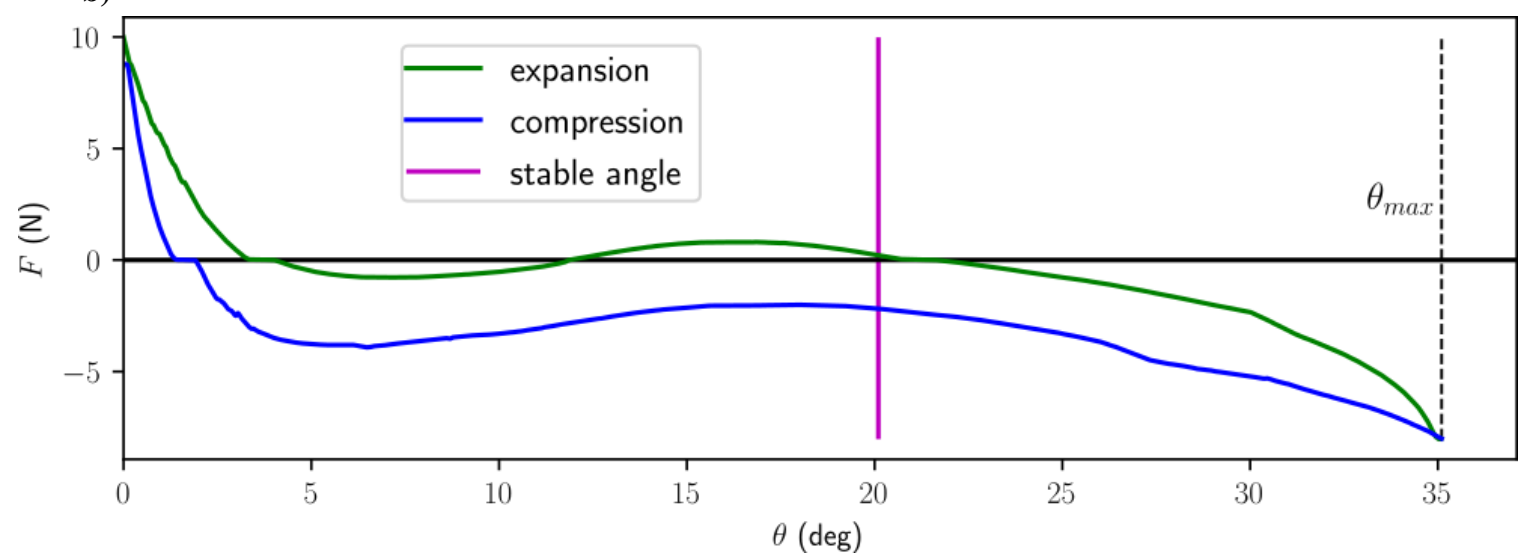

Fig. 10: a) Use of the test bench with the custom-made sliding mechanical support, allowing the origami structure to bend while recording the force. b) Force curves. Expansion (in green) and compression/bending (in blue). Parameters: $R=40 \mathrm{~mm}$, $\rho=0.4, \alpha_{1}=45$ and $\alpha_{2}=30$. It is worth noting that the small distortions on the graph when the forces are close to zero correspond to backlash produced by a small gap on the sliding mechanical support. 